\title{
Connecting the density structure of molecular clouds with star formation
}

\author{
Jouni Kainulainen \\ Max-Planck-Institute for Astronomy, \\ Königstuhl 17, 69117 Heidelberg \\ email: jtkainul@mia.de
}

\begin{abstract}
In the current paradigm of turbulence-regulated interstellar medium (ISM), star formation rates of entire galaxies are intricately linked to the density structure of the individual molecular clouds. This density structure is essentially encapsulated in the probability distribution function of volume densities ( $\rho$-PDF), which directly affects the star formation rates predicted by analytic models. Contrasting its fundamental role, the $\rho$-PDF function has remained virtually unconstrained by observations. I describe in this contribution the recent progress in attaining observational constraints for the column density PDFs ( $N$-PDFs) of molecular clouds that function as a proxy of the $\rho$-PDFs. Specifically, observational works point towards a universal correlation between the shape of the $N$-PDFs and star formation activity in molecular clouds. The correlation is in place from the scales of a parsec up to the scales of entire galaxies, making it a fundamental, global link between the ISM structure and star formation.
\end{abstract}

Keywords. stars: formation, ISM: clouds, ISM: structure

\section{Introduction}

How exactly different physical processes give rise to the star formation rates of molecular clouds is a decades old open question. Most of all, we do not understand how the different processes mould the structure and dynamics of the clouds, causing only a small fraction of their gas to have conditions suitable for star formation. Observationally, we know very well that the star formation rates and efficiencies of molecular clouds depend strongly on the internal structure of the clouds (e.g., Kainulainen et al. 2009, 2014; Lada et al. 2012; Evans et al. 2014). Therefore, the first step in understanding the regulation of star formation is understanding the regulation of the internal cloud structure.

The major analytic theories that predict star formation rates in the ISM encapsulate the information about the internal cloud structure in the probability density function of gas densities $(\rho$-PDF). The function describes the probability of a unit volume to have a certain density. The function is a basic ingredient in virtually all commonly used analytic theories that start from the basic physical processes acting in the ISM (reviewed in Padoan et al. 2014, see also Federrath \& Klessen 2012). Recent studies have also established that the distributions of column densities $(N$-PDFs) can carry the same information as the $\rho$-PDFs (e.g., Brunt et al. 2010; Federrath \& Klessen 2013; Kainulainen et al. 2014), enabling their use as observationally reachable proxies of the $\rho$-PDFs.

The missing piece of the star formation rate models is that the $\rho$-PDF function has not been observationally well constrained. And by lack of observational constraints, the models have turned to numerical simulations for information about it. These simulations have heavily concentrated in modelling supersonic turbulence, including also other physics (self-gravity, magnetic fields, chemistry) as computational capabilities have begun to allow it. The basic result of the simulations is that isothermal, supersonic, driven turbulence develops a lognormal $\rho$-PDF (e.g., Vázquez-Semadeni 1994; Federrath et al. 
2010; Molina et al. 2012). Consequently, the star formation rate theories commonly adopt this framework and with it the lognormal $\rho$-PDF. This means that the key ingredient of the models remains not confronted by systematic observations.

\section{Universal link between the $N$-PDFs and star formation?}

Observational works are now in progress of changing this picture; they are beginning to provide systematic constraints for the column density statistics of molecular clouds. This progress has been made possible by the novel dust extinction mapping techniques in near-infrared and Herschel dust emission measurements in far-infrared and sub-mm (e.g., Lombardi 2009; André et al. 2010). These techniques use dust to trace the column density distributions of the clouds and can provide high-sensitivity maps in resolution that reaches some $0.05 \mathrm{pc}$ in the Solar neighbourhood clouds. Such resolution means that the resulting maps contain hundreds of thousands of independent resolution elements, enabling studies of the column density statistics.

The above techniques have already given us a basic picture of the $N$-PDFs. Now more than six years ago, we performed the first systematic survey of the $N$-PDFs in the Solar neighbourhood clouds (Kainulainen et al. 2009). In this work, we analysed dust extinction-derived column density maps of practically all clouds closer than 260 pc to the Sun. This work gave rise to two important results. First, we showed that the $N$-PDFs of most clouds are poorly fitted with log-normal functions when considering their entire column density range. Rather, they showed a possibly log-normal shape only at low-column densities $\left(N(\mathrm{H})<4-6 \times 10^{21} \mathrm{~cm}^{-2}\right)$, while higher column densities showed a significant, power-law like excess to that shape. This was an important result, as it seemed to at least partially contradict the prediction adopted from the turbulenceregulated ISM framework that molecular clouds might carry log-normal $\rho$-PDFs (and $N$-PDFs, e.g., Federrath \& Klessen 2013).

The second important result from Kainulainen et al. (2009) was that the $N$-PDFs showed a variety of shapes that correlated with the number of young stars in the clouds. Specifically, clouds with more young stellar objects (YSOs) showed more top-heavy $N$ PDFs than those with less YSOs. In the extreme of this relation, the couple of clouds in the sample that had no young stars within had very bottom-heavy, close-to log-normal $N$-PDFs. We quantified this trend better later by showing that the mean star formation rate per unit area in entire molecular clouds correlates with the top-heaviness of their $\rho$-PDFs (Kainulainen et al. 2014).

Studies employing Herschel data have found the same trend between the star formation activity and the shape of the $N$-PDFs (e.g., Schneider et al. 2013; Alves de Oliveira et al. 2014). They have also raised a question whether the $N$-PDFs of any clouds are log-normal, or are all $N$-PDFs better described by power-laws (Lombardi et al. 2015). The first Herschel studies have also reached outside the Solar neighbourhood, towards more massive molecular clouds in the Galaxy (e.g., Schneider et al. 2015).

One great advantage of the high resolution of the Herschel data is that it allows us to zoom in and study the $N$-PDFs within molecular clouds in the Solar neighbourhood (e.g., Stutz \& Kainulainen 2015, see Fig. 1). This allows linking the $N$-PDFs not only to the mean star formation activity of the clouds, but also to their local star formation rates. This is possible, because the protostars are identifiable in the Solar neighbourhood clouds, e.g., using Herschel data and/or near- and mid-infrared colour selections. The first works taking advantage of this have found that in parsec-scale regions within clouds the number of Class 0 protostars per unit area correlates with the top-heaviness of the 

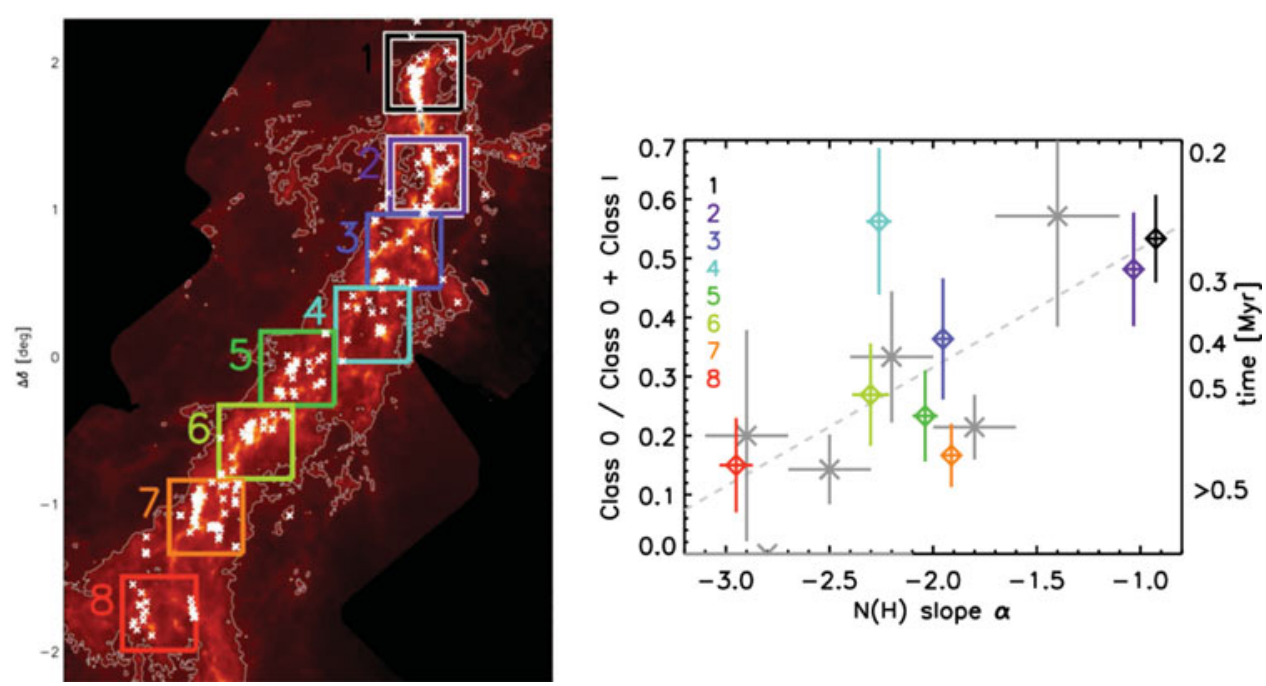

Figure 1. Left: Column density map of Orion A derived using Herschel observations (Stutz \& Kainulainen 2015). The white crosses show the protostars in the cloud. The boxes show the eight regions in which the relation between protostars and $N$-PDFs was studied. Right: Class 0 protostar fraction as a function of the $N$-PDF power-law slope in the eight regions within Orion A (see the left panel). The y-axis on the right gives the evolutionary time-scale assuming a constant star formation rate. The grey data points are for the Perseus cloud (Sadavoy 2013; Sadavoy et al. 2014).

N-PDFs (Sadavoy et al. 2014; Stutz \& Kainulainen 2015). This means that at these scales the on-going star formation rate activity is linked to the local gas mass distribution.

Intriguingly, the first small-scale studies have found that the evolutionary time-scale of parsec-scale regions inferred from Class 0 and 1 protostars anti-correlates with the top-heaviness of the $N$-PDFs (e.g., Stutz \& Kainulainen 2015). In other words, regions with more top-heavy $N$-PDFs have shorter protostellar time-scales. This is curious, as it suggests that regions with flatter $N$-PDFs may be younger in terms of their recent star formation. Such a result is not trivial to interpret in the light of current models for $N$-PDF evolution that predict evolution from a bottom-heavy, turbulence-dominated state to a top-heavy, gravity-dominated state (e.g., Federrath \& Klessen 2013; Girichidis et al. 2014; Ward et al. 2014).

Is the strong relationship between star formation and density distributions also prevalent at Galactic scales? The answer seems to be yes. Abreu-Vicente et al. (2015) performed the first systematic Galactic scale study of the $N$-PDFs, analysing 330 molecular clouds using the ATLASGAL dust emission survey (Fig. 2). The main result of this work was that indeed, objects that showed no signs of on-going star formation had the most bottom-heavy $N$-PDFs. Star-forming clouds that harbour protostars had more top-heavy $N$-PDFs, and finally, the most active clouds that contained HII regions had the most top-heavy $N$-PDFs (Fig. 2). This finding showed that the correlation between the $N$ PDFs and star formation exists also at Galactic scales; therefore, it must represent a fundamentally important relation describing star formation in the ISM.

Finally, indications of a similar trend have also been detected in external galaxies. Using interferometic observations of CO emission from the entire M51 galaxy, Hughes et al. (2013) showed that regions that had higher surface density of star clusters also showed more top-heavy $N$-PDFs. These observations again evidence a fundamental correlation between the column density structure and star formation activity. 

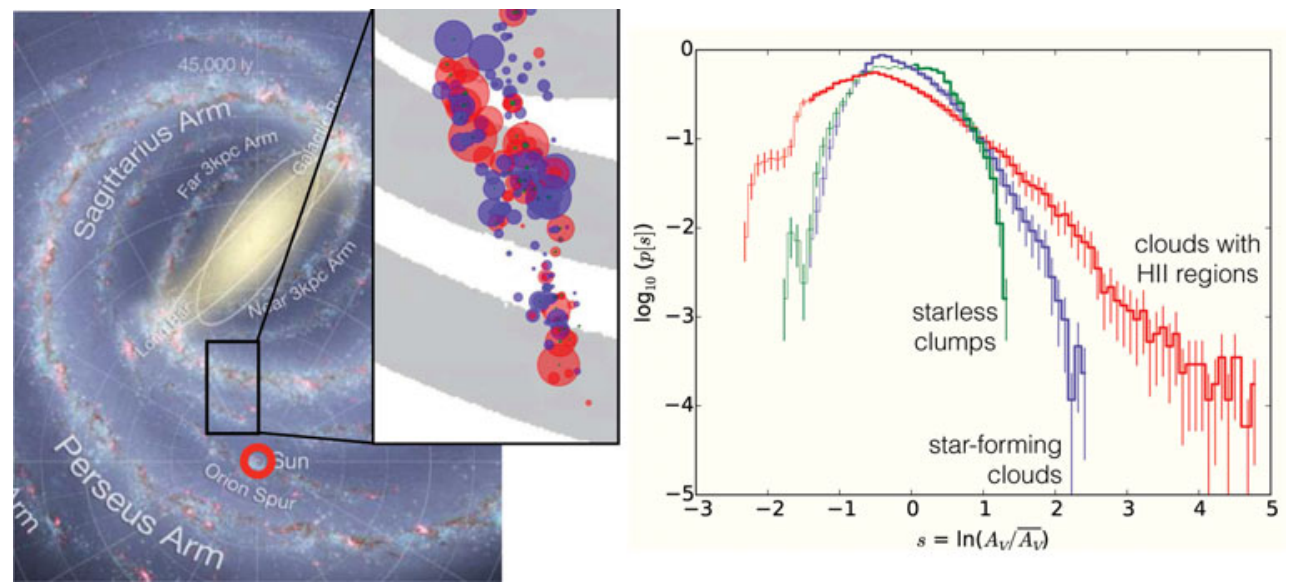

Figure 2. Left: The first Galactic-scale study of the molecular cloud N-PDFs (Abreu-Vicente et al. 2015, partial credit: NASA/JPL-Caltech/R. Hurt, SSC-Caltech). The red, blue, and green colours refer to clouds containing HII regions, star-forming clouds, and starless clumps. Right: The total $N$-PDFs of each object class. The starless clumps have the most bottom-heavy $N$-PDFs and the clouds containing HII regions the most top-heavy $N$-PDFs.

While the last half a decade has seen an entirely new set of observational constraints for the column density statistics of the Solar neighbourhood clouds, works currently in progress are expected to extend that set to Galactic volumes. Such an observational view can confront the star formation theories, and the entire turbulence-regulated ISM framework, at the scales relevant for Galactic star formation.

\section{References}

Abreu-Vicente, J., Kainulainen, J., Stutz, A., Henning, T., \& Beuther, H. 2015, A\&AA, 581, A74 Alves de Oliveira, C., Schneider, N., Merín, B., et al. 2014, A\&A A, 568, A98

André, P., Men'shchikov, A., Bontemps, S., et al. 2010, A\&SA, 518, L102

Brunt, C. M., Federrath, C., \& Price, D. J. 2010, MNRAS, 405, L56

Evans, N. J., II, Heiderman, A., \& Vutisalchavakul, N. 2014, ApJ, 782, 114

Federrath, C. \& Klessen, R. S. 2013, ApJ, 763, 51

Federrath, C. \& Klessen, R. S. 2012, ApJ, 761, 156

Federrath, C., Roman-Duval, J., Klessen, R. S., et al. 2010, A\&\&A, 512, A81

Girichidis, P., Konstandin, L., Whitworth, A. P., \& Klessen, R. S. 2014, ApJ, 781, 91

Kainulainen, J., Federrath, C., \& Henning, T. 2014, Science, 344, 183

Kainulainen, J., Beuther, H., Henning, T., \& Plume, R. 2009, A\&A, 508, L35

Lada, C. J., Forbrich, J., Lombardi, M., \& Alves, J. F. 2012, ApJ, 745, 190

Lombardi, M. 2009, A\& $A, 493,735$

Lombardi, M., Alves, J., \& Lada, C. J. 2015, A\&A, 576, L1

Molina, F. Z., Glover, S. C. O., Federrath, C., \& Klessen, R. S. 2012, MNRAS, 423, 2680

Padoan, P., Federrath, C., Chabrier, G., et al. 2014, in: H. Beuther, R. Klessen, C. Dullemond, T. Henning (eds.), Protostars and Planets VI, p. 77

Sadavoy, S. 2013, PhD Thesis, University of Victoria.

Sadavoy, S. I., Di Francesco, J., André, P., et al. 2014, ApJL, 787, L18

Schneider, N., André, P., Könyves, V., et al. 2013, ApjL, 766, L17

Schneider, N., Csengeri, T., Klessen, R. S., et al. 2015, A\&A, 578, A29

Stutz, A. M. \& Kainulainen, J. 2015, A\& A, 577, L6

Vázquez-Semadeni, E. 1994, ApJ, 423, 681

Ward, R. L., Wadsley, J., \& Sills, A. 2014, MNRAS, 445, 1575 